\title{
The secret of eternal youth in the Energetic Forest The study of the Energetic Forest through market and customer psychology.
}

\author{
Shiqi Feng ${ }^{1}$ \\ ${ }^{1}$ Long Island Lutheran High School 131 Brookville Road NY 11545
}

\begin{abstract}
The Chinese beverage brand Energetic Forest experienced great success in the last few years with its release of 0 sugar soda drinks. Its secret for success lies in its three marketing strategies and one core concept of its product. The first is to adopt a Japanese style package of the bottles of the products. The second is to focus on convenience stores instead of large supermarkets. The third is to utilize celebrity fame and social media accounts to popularize its products. Its idea to promote healthy and delicious drinks is very attractive to its target customers in China. The success in its strategies is inseparable from the change in young generation's life styles caused by the advancement of technology and progress in China's economy.
\end{abstract}

\section{Introduction}

Nowadays, China's beverage market is mainly divided into three areas: the first is the traditional Chinese beverage brands, such as Master Kong and Wahaha; the second is the revival of old brands, such as Arctic Ocean; the third is the traditional international beverage brands, such as Coca Cola and Pepsi. Energetic Forest focuses on 0 sugar, 0 fat and 0 calorie. This is not a new market, but Energetic Forest has an outstanding performance.

The drinks launched by Energetic Forest are a series of popular drinks in the Chinese market recently. The main products are all located in the well-known beverage categories, because the big category has a big market, and only the categories that people are already familiar with do not need to pay the expensive cost of education.

However, to enter the mature category, how to avoid the quagmire of homogeneous competition, how to avoid falling into the low-level competitive environment of channel competition, price competition and advertising resource competition, and how to obtain breakthrough opportunities in the competition through the creation of brand value need to carefully plan the marketing strategy in combination with the advantages of the enterprise itself. Obviously, the Energetic Forest has performed very well.

According to media reports, the sales volume of Energetic Forest brand drinks exceeded 800 million yuan in the first half of 2020, and the sales volume in May alone reached 260 million yuan, which exceeded the total sales of the whole year in 2018. The sales volume of Tmall's 618 Shopping Festival surpassed Coca Cola and ranked first in the list of water drinks. [1] Although a young brand, Energetic Forest has acquired astonishing sales records in the last few years.

\section{Advantages}

Ordinary sparkling water is generally colorless and tasteless, and the representative brands, Perrier and S. Pellegrino, are relatively expensive. Their sparkling water is expensive and not as good as ordinary soda drinks. These reasons prevent people from finding healthy alternatives. Obviously, Energetic Forest has seized the opportunity. In my opinion, the success of Energetic Forest is divided into three parts. 1) it makes use of domestic consumers' love for Japanese products 2) it depends on the convenience stores at which more and more domestic consumers are shopping instead of on large supermarkets 3) it uses female consumers' frequent patronage of social media app and celebrity effect to market its products.

\subsection{Brand Packaging}

Define abbreviations and acronyms the first time they are used in the text, even after they have been defined in the abstract. Energetic Forest chose to package its own brand into a Japanese brand. Energetic Forest is actually a company registered in Beijing, and all aspects of its production and design are also completed in China. Why, you may wonder, is it packaged as a Japanese brand? For one thing, Chinese people have a strange and familiar feeling to Japan and Korea because of their geographical proximity to China, and for another, the minimalist style of Japanese products has made many people feel good, which can be seen from the popularity of MUJI and Uniqlo in China.

In MUJI shops, it is difficult to find well-packaged 
products. Make up water is a transparent bottle packaging, clothing is the simplest style, there is no eye-catching gorgeous color, there is no stay in the strange shape, all simple, back to the original. Its design pursues the maximum value of the thing itself rather than give additional functions to the commodity. However, MUJI design is not just simple. There are profound intellectual, perceptual and sufficient sense of personal design and high-tech in simplicity. Represented by MUJI, people gradually formed an impression of Japanese products which are concise but rich in functions, excellent in quality and personalized, so they are particularly willing to purchase Japanese products.

It is undeniable that with the development of the Internet, international culture is spreading faster. Many Japanese cultures can also be exchanged to China. Many young people like Japanese comics and anime very much, so they tend to buy Japanese brands. And more than $88 \%$ of the Energetic Forest's customer base is young people. Therefore, packaging the brand into a Japanese brand is a very clever way, which undoubtedly appeals to more customers' tastes and to more potential customer groups.

Therefore, Energetic Forest found the Chinese consumers' interests and worked hard to achieve that expectation. It directly packaged itself into a Japanese brand.

The product used traditional Chinese characters instead of simplified characters and printed the "Supervised by Nippon Corporation" logo, making everyone feel that this is a Japanese brand. In order to get this certificate, Energetic Forest has even registered a company in Japan. The major products are trying the best to imitate famous Japanese brands' styles. For example, the big doll pattern on the milk tea packaging imitates the Japanese Fujiya, and its burning tea imitates the packaging of Japanese LUCIPIA products, thus allowing itself to successfully become consumers' favorite. Consumers who saw the familiar style, coupled with its 0 sugar, 0 fat, and 0 calorie logo, will tend to buy the product. When fitness became a necessary condition for all young middle-class people in China, Healthy drinks instantly fit everyone's lifestyle. This is an important strategy that Energetic Forest chooses.

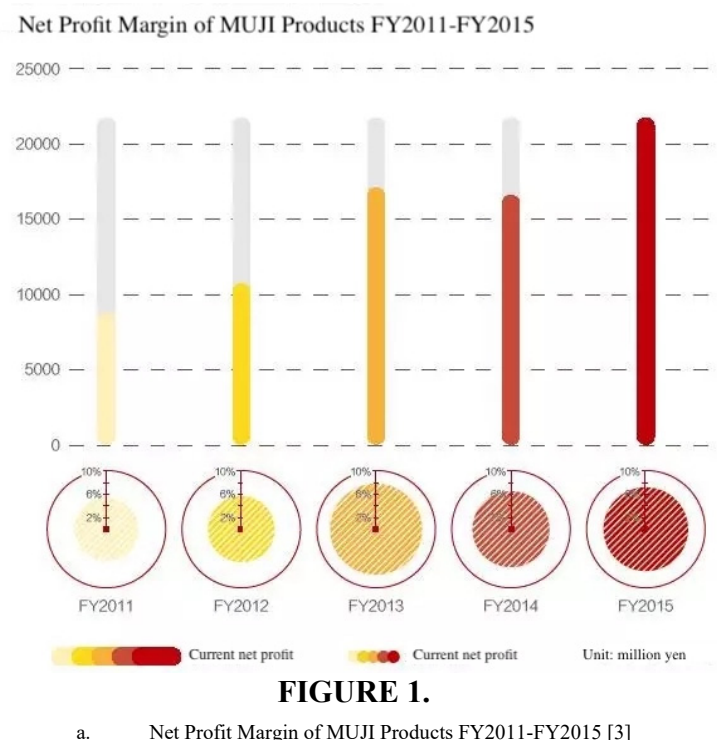

\subsection{Convenience Stores}

Its second strategy is to spread brand awareness through convenience stores. According to the list of "China's top 100 chain stores in 2018" released by the China Chain Management Association, [2] among the top 100 chain stores, the growth rate of convenience stores is far ahead: the sales scale of top 100 convenience stores enterprises increased by $21.1 \%$ year-on-year, the number of stores increased by $18.0 \%$, and 11944 new stores were added, accounting for $62.5 \%$ of the total number of new stores. In contrast, the sales volume of the top 100 enterprises mainly operating large supermarkets increased by $2.5 \%$ on average and the number of stores increased by $3.6 \%$, which was significantly lower than the average growth rate of the top 100 enterprises.

At present, the pace of life is faster and faster, and China is a country with high population density. People are in great need of a convenient and fast place, where convenience stores can replace the department stores or supermarkets in the past. Especially for the young people in the office area, they usually want to have a quick lunch. The convenience store is a good place for them to go. The Energetic Forest has come to the young people's insight since it was launched.

And online shopping mall is also the shopping choice of most young people, because the logistics is very convenient and fast. Immediately, it has achieved comprehensive cooperation with Tmall. With the help of e-commerce, another gathering place for young people, Energetic Forest has successfully publicized its products.

\subsection{Marketing}

The third important strategy is to apply large-scale marketing, a method different from traditional marketing. There are two main marketing positions of Energetic Forest. One is Xiaohongshu, an app designed to recommend useful and interesting things in life to people. Energetic Forest mainly focuses on social marketing ideas. First, the brand stands out through Weibo, and in the later time, when there is a certain customer base, they started to crystalize targeted customers. Young women. According to incomplete statistics, Energetic Forest has more than 5000 notes in Xiaohongshu, far more than Perrier (more than 3000 articles), Oriental leaves (more than 1600 articles), and Hankou No.2 factory (more than 700). At the same time, Energetic Forest focused on the celebrity effect. They have found the most popular stars at the moment, most of them are young male stars, hoping to attract the attention of young women.

Moreover, Energetic Forest chose an unexpected way. They decided to advertise on the elevator. The mandatory contact of elevator media makes people have to see it every day. Fast but easy to remember advertising words and product features have brought huge impact to consumers. It only takes a few days to remember the brand. Similarly, on other popular social media platforms, such as Tiktok, Bilibili, and Weibo, they will find people to promote their products. For a long time, people kept seeing this brand appearing in their lives, so they would naturally be curious and want to buy its products. 
The success of the Energetic Forest is not only the role of marketing but also must have a certain product capability support. Further analysis can find three more accurate focuses, 1. Insight into trends and concept creation must be based on new product force; 2 . multifaceted potential and even detonating must be based on the innovative channel touch and user interaction mode; 3 Large scale replication of capital entry must be based on the considerable growth data in the early stage. Successful entrepreneurs have their own micro innovation in different segments.

Grasping the consumption trend and creating new concepts is the first step in the rise of new consumer brands. Therefore, many people simply attribute the success of the founders' Tang Binsen and Energetic Forest to their accurate grasp of young people's pursuit of sugarfree drinks and a healthy lifestyle. However, if carefully considered, this reason is not very tenable. In other words, this factor alone is not enough to lead to the success of the Energetic Forest.

\section{Sugar-free}

The sugar-free beverage is not the patent invention of the Energetic Forest. As early as 2011, Nongfu Spring launched the sugar-free tea drink "Oriental Leaves". In 2012, Master Kong also launched sugar-free tea drink. On the track of sugar-free carbonated drinks, Coca Cola launched diet coke in the 20th century; in the field of fruit bubble water, Perrier also launched several kinds of sugarfree bubble water in fruit flavor. Therefore, the success of the Energetic Forest is not due to its first launch of some kind of products with absolute advantages. However, in terms of product power, the Energetic Forest has optimized itself to the position of top brand of the market.

The concept of sugar-free drinks is a very beneficial attempt for many brands. First, healthy drinks will not only help wash away the bad reputation of producing junk food but also reshape the high-quality brand image that conforms to the call of the times. Secondly, introduce new growth points and add merit to the development of the brand helped it get greater support in the capital market. Third, healthy drinks also appeal to customers, making them willing to understand the overall brand and then enhance the sales of traditional products. In fact, if these new products are properly developed, customers will understand the overall brand, increasing the sales of the company's traditional high-calorie and high-fat products. That is to say, under the strategy of product line expansion, it is very likely that after attracting a number of new consumers through sugar-free product, it will, in turn, promote the sales growth of original product [4].

Sugar-free drinks were not the first to be created, but the Energetic Forest has fixed the shortcomings of the previous generation:

1. the sugar-free tea drink launched earlier may be healthier, but the biggest problem is that there is no sweet taste. Besides the sweet drinks such as sugar-free tea, Oriental Leaves, sugar-free Perrier water, consumers are limited to be a small group. They are highly concerned about their health and willing to bear taste loss. This is also an important reason for the sweet taste of Energetic Forest tea drinks: because the Energetic Forest hopes to acquire a larger customer base -those who are not willing to pay for their health can also choose their brand.

2. Coca-Cola and Pepsi do have a large number of sugar-free carbonated products, but most of their products, including coke, Fanta, and Sprite, have been branded as unhealthy by most people as a stereotype, which is hard to reverse.

From the perspective of resource strength, the Energetic Forest falls far behind Coca-Cola and Pepsi, and it has no ability to invent new sweeteners. However, this does not prevent its single breakthrough in publicity - it claims that it has found "Erythritol" - a sucrose substitute which can guarantee the taste of sweet taste and avoid a large amount of heat. Unlike Aspartame added to sugarfree coke, Erythritol has no side effects on health. This is one of the key reasons why the Energetic Forest stands out. Since Energetic Forest's advertisements always promote the healthy quality of its drinks, people will naturally prefer to buy healthier drinks.

The staff of Energetic Forest once said, "All new products of Energetic forest come from big data, whether it's packaging, product naming, or product taste. All of them will not be transferred by anyone's personal will after they are determined." This is the fundamental reason why Energetic Forest can be so famous. From the big data analysis, we can understand what the new generation of consumers are like. And from positioning to products to taste to packaging, what consumers like subconsciously, they will work in any direction. In China, the so-called female users win the world.

\section{Conclusion}

Carbonated drinks are so popular not only because of their sweet taste but also because it contains soda. Ordinary sparkling water is generally colorless and tasteless, and the representative brands' Perrier and S. Pellegrino are relatively expensive. These sparkling water drinks are expensive and not as good as ordinary soda drinks. These reasons prevent people from finding healthier alternatives. Obviously, Energetic Forest has seized the opportunity.

The Energetic Forest's breakthrough in sales is mainly due to its three sales strategies. The first is to package the brand into a Japanese brand. The second is to take the convenience stores and Internet commonly used by young people as the main sales channels. Third, its use of major social media software and star effect to promote their products. Although it is not the first one to make the concept of sugar free, it uses the concept of sugar-free to make the product very successful.

Through its three strategies, Energetic Forest has successfully won the unexpected customer group of young women, and gradually expanded its popularity and products' exposure. 


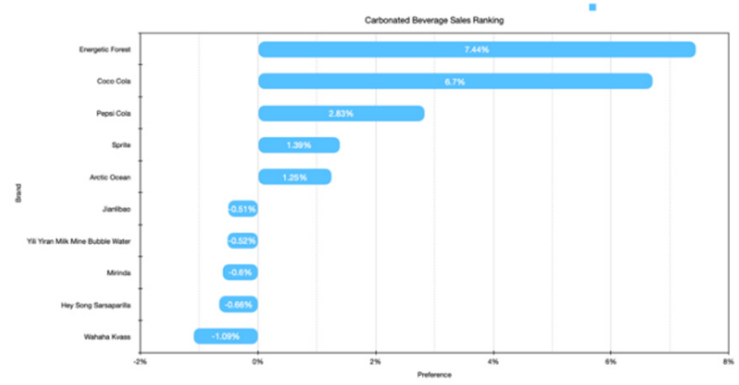

FIGURE 2.

a. Sales of the Energetic Forest. [5]

\section{References}

1. Wang, Guanxiong. "Four Year Sales of 1 Billion Bottles and Valuation of 10 Billion. Here Is the Secret of the Energetic Forest!" Sohu, 3 Aug. 2020, 15:52, www.sohu.com/a/411223691_115389.

2. Ccfa, China Chain Store \& Franchise Association. "Top 100 Chinese Chain Stores in 2018." China Chain Store \& Franchise Association, China Chain Store \& Franchise Association, 9 May 2019, www.ccfa.org.cn/portal/cn/view.jsp?lt=31\&id=4386 36.

3. Yuan, Jingying. "Secret of MUJI's Annual Sales of 20 Billion: Only Serve 10\% of The Population." Link Shop, Link Shop, 26 Nov. 2016, 10:16, www.linkshop.com.cn/web/archives/2016/364696.sh tml.

4. Grierson, Jamie. "Coca-Cola Says Sugar Cuts Have Not Harmed Sales." The Guardian, Guardian News and Media, 17 May 2017, www.theguardian.com/society/2017/may/17/cocacola-says-sugar-cuts-have-not-harmed-sales.

5. Rufei, Yao. "'Double 11': Has 'Generation Z' Become the Mainstream of Consumption, with Turnover Exceeding 400 Billion?" Wangyi, Zhongxinjingwei, 20 Oct. 2020, 12:00:13, www.163.com/dy/article/FPCN0LRM0519C6T9.ht $\mathrm{ml}$. 